\title{
Efecto del ciprofibrato sobre el metabolismo del colesterol HDL y la capacidad antioxidante plasmática en el ratón
}

\author{
Camila Mendoza ${ }^{1}$, Verónica Quiñones ${ }^{1}$, Ludwig Amigo ${ }^{2}$, Dolores Busso ${ }^{1}$, \\ Alberto Maiz ${ }^{1}$, Attilio Rigotti. ${ }^{1,3}$ \\ 1. Departamento de Nutrición, Diabetes y Metabolismo \\ 2. Departamento de Gastroenterología \\ 3. Centro de Nutrición Molecular y Enfermedades Crónicas. \\ Pontificia Universidad Católica de Chile. \\ *Financiamiento: Proyecto FONDECYT Regular N¹110712
}

Antecedentes: Las dislipidemias, ya sea un aumento en los niveles de colesterol LDL y/o una disminución en las cifras de colesterol HDL, son muy relevantes para el desarrollo de la enfermedad cardiovascular ateroesclerótica, siendo el colesterol HDL bajo la dislipidemia más frecuente en la población chilena. Con respecto al colesterol HDL bajo y los triglicéridos elevados, los fibratos, agonistas del receptor nuclear PPAR- $\alpha$ que modula la transcripción de genes involucrados en el metabolismo de lípidos, representan una importante alternativa de manejo farmacológico de las dislipidemias. Sin embargo, estudios clínicos recientes no han sido concluyentes con respecto a su beneficio real sobre el control de la ateroesclerosis cuando se usan combinados con estatinas.

Objetivo: Evaluar el impacto de la administración de fibratos sobre el metabolismo del colesterol HDL y la función antioxidante del plasma usando el ratón como modelo experimental.

Metodología: Los ratones de la cepa C57BL/6 fueron tratados con ciprofibrato al $0,2 \%$ en dieta control durante 7 días. Luego del tratamiento, se analizaron los niveles de colesterol plasmático y triglicéridos, la expresión hepática de proteínas claves involucradas en el metabolismo de colesterol HDL, el contenido de colesterol hepático, la secreción de colesterol biliar y el daño oxidativo y la función antioxidante plasmática. Resultados: El tratamiento con ciprofibrato disminuyó significativamente los niveles de triglicéridos plasmáticos y la expresión hepática del receptor de HDL SR-BI, efecto que se correlacionó con un aumento en el tamaño de las partículas de HDL, pero no en los niveles de colesterol HDL. Además, el ciprofibrato disminuyó los niveles proteicos de los transportadores de colesterol ABCG1 y ABCG8, aunque no modificó $\mathrm{ABCA} 1$, en conjunto con una reducción del contenido hepático de colesterol y un aumento en la secreción de colesterol hacia la bilis. Finalmente, el uso de este hipolipemiante mejoró la función antioxidante del plasma, aunque se detectó un aumento en el daño nitrosativo de las proteínas plasmáticas.

Conclusión: Este estudio ha permitido obtener nueva información sobre el efecto metabólico y funcional de la administración de fibratos en ratones, lo cual podría ayudar comprender los resultados de estudios clínicos recientes que han usado esta clase de hipolipemiantes en humanos.

\section{Correspondencia:}

Dr. Attilio Rigotti,

Departamento de Nutrición, Diabetes y Metabolismo,

Escuela de Medicina, Pontificia Universidad Católica de Chile.

Marcoleta \#367 (interior) $4^{\circ}$ Piso, Santiago, 8330024, Chile.

Teléfono: 56-2-23543832.

arigotti@med.puc.cl 


\section{Effect of ciprofibrate on HDL cholesterol metabolism and plasma anti-oxidant capacity in mice}

Background: Increased serum levels of LDL cholesterol and/or decreased values of HDL cholesterol are very relevant for atherosclerotic cardiovascular disease. Low HDL cholesterol is the most prevalent dyslipidemia in the Chilean population. Regarding reduced HDL cholesterol and high triglyceride levels, fibrates, nuclear receptor PPAR- $\alpha$ agonists that modulate transcription of genes involved in lipid metabolism, represent an important alternative for pharmacological management of dyslipidemia. However, recent clinical studies have been inconclusive with respect to their real benefit on atherosclerosis when used in combination with statins.

Aim: To evaluate the impact of fibrate administration on HDL cholesterol metabolism and antioxidant plasma functionality using the mouse as experimental model.

Methodology: Using wild-type C57BL/6 mice, ciprofibrate was administered at $0.2 \%$ in chow diet for 7 days. After treatment, plasma cholesterol and triglycerides levels, hepatic expression of key proteins involved in HDL cholesterol metabolism, liver cholesterol content, biliary cholesterol secretion, and plasma oxidative damage and antioxidant function were analyzed.

Results: Ciprofibrate treatment significantly decreased plasma triglycerides levels and hepatic HDL receptor SR-BI expression. This latter finding was associated with increased HDL particle size, without changes in HDL cholesterol levels. Furthermore, ciprofibrate decreased hepatic expression of cholesterol transporters $\mathrm{ABCG} 1$ and $\mathrm{ABCG} 8$, but not $\mathrm{ABCA} 1$, which correlated with reduced liver cholesterol content and increased biliary cholesterol secretion. Finally, fibrate therapy improved plasma antioxidant function, even though increased nitrosative plasma protein damage was detected.

Conclusion: This study has provided new information on metabolic and functional effects derived from fibrate use in mice and it may help to better understand recent clinical findings using this lipid-lowering drug class in humans.

Keywords: HDL cholesterol; HDL functionality; Atherosclerosis; PPAR- $\alpha$; ciprofibrate 
Introducción: Debido a la actual transición epidemiológica, las enfermedades crónicas no transmisibles, incluyendo la ateroesclerosis, constituyen la causa más común de morbi-mortalidad en el mundo ${ }^{1-2}$. La patogénesis de la ateroesclerosis, cuyas complicaciones cardiovasculares isquémicas representan la principal causa de muerte en Chile y el mundo, es multifactorial y modulada por diversos factores de riesgo no modificables y modificables. Dentro de estos últimos, la presencia de niveles elevados de colesterol LDL (c-LDL) es un factor de riesgo cardiovascular muy prevalente y relevante en la etiopatogenia de la enfermedad cardiovascular de origen aterosclerótico ${ }^{3}$. Por otro lado, la evidencia clínica indica que la disminución del c-LDL mediante el uso de estatinas determina una reducción en la incidencia de eventos cardiovasculares isquémicos ${ }^{4}$. Sin embargo, a pesar de un óptimo control del c-LDL, más del $50 \%$ de los pacientes que usan estatinas aún presentan riesgo de desarrollar eventos isquémicos coronarios y cerebrovasculares, destacando la necesidad de implementar otras aproximaciones terapéuticas para disminuir este riesgo cardiovascular residual.

En relación a otros factores de riesgo, los niveles reducidos de colesterol HDL (c-HDL) representan un predictor independiente y significativo de enfermedad cardiovascular ateroesclerótica, mientras que altos niveles de c-HDL se consideran como un factor ateroprotector y de mejor pronóstico cardiovascular-8. ${ }^{5-8}$ La epidemia de obesidad, síndrome metabólico y diabetes han aumentado la prevalencia de c-HDL bajo como factor de riesgo para ateroesclerosis. Más aún, el c-HDL bajo es la dislipidemia más prevalente en varias regiones del mundo, incluyendo Latinoamérica. En Chile, la Encuesta Nacional de Salud (ENS) del año 2009-2010 mostró que el c-HDL bajo es la dislipidemia más frecuente, con una prevalencia global de $45 \%$ en la población adulta chilena 9 .

Las partículas de HDL, un subgrupo heterogéneo de las lipoproteínas circulantes, actúan captando colesterol libre desde los tejidos periféricos y -tras su transformación hacia ésteres de colesterol en la circulación- lo transportan hacia el hígado y los tejidos esteroidogénicos en forma directa por vía del receptor de HDL SR-BI (receptor scavenger clase $\mathrm{B}$ tipo I) ${ }^{10} \mathrm{o}$ en forma indirecta por su traspaso hacia las lipoproteínas no HDL seguido de la captación y el catabolismo de estas últimas a nivel hepático. La excreción del colesterol desde el hígado hacia la bilis completa el proceso conocido como "transporte reverso de colesterol", el cual representaría uno de los mecanismos más importantes que explican el efecto ateroprotector de las HDLs ${ }^{11}$. La evidencia epidemiológica en humanos aún es ambigua en relación a la expresión de SR-BI, los niveles de HDL y el riesgo cardiovascular, sin embargo, una reciente publicación de Zanoni y cols. ${ }^{12}$ identificó en SCARBI, el gen que codifica SR-BI, la variante P376L, que altera la expresión del receptor en superficie y que tiene como consecuencia, que los individuos presenten niveles plasmáticos elevados de c-HDL y, a pesar de esto, mayor riesgo cardiovascular. Esto refuerza la necesidad de validar ensayos que evalúen la función de las HDL más allá de la medición estática de los niveles de c-HDL, pues las HDL también participan y regulan otros procesos biológicos, tales como la hemostasis dependiente de las plaquetas, la apoptosis, el estrés oxidativo, el metabolismo de la glucosa y la inmunidad innata ${ }^{13-15}$, de modo que sus propiedades bioactivas no queden definidas únicamente en términos de los niveles plasmáticos, sino que evaluando su función en relación al efecto protector sobre la ateroesclerosis y otras enfermedades crónicas. Por lo anteriormente mencionado, la modulación de los niveles y/o la funcionalidad de las lipoproteínas HDL representan una alternativa de blanco terapéutico farmacológico y no farmacológico para un mejor control del riesgo cardiovascular residual que persiste después del uso de estatinas.

Los fibratos son drogas hipolipemiantes que modulan el metabolismo lipídico por activación del receptor nuclear PPAR- $\alpha$ (receptores activados por proliferadores perixosomales tipo alfa), el cual regula la transcripción de genes involucrados en el metabolismo de los ácidos grasos, el colesterol y las lipoproteínas ${ }^{16}$. La activación de PPAR- $\alpha$ estimula la $\beta$-oxidación de ácidos grasos en el hígado, reduciendo su disponibilidad para la síntesis y la secreción de VLDL, aumenta la expresión de la lipoproteína lipasa (LPL), estimulando la hidrólisis de lipoproteínas ricas en triglicéridos, y disminuye la expresión de apolipoproteína CIII, un factor inhibidor de la actividad de LPL, todas funciones que tienen como consecuencia global una disminución en la síntesis y un aumento en el remodelamiento de las lipoproteínas no HDL y una disminución en los niveles plasmáticos de triglicéridos ${ }^{16-20}$. Por otro lado, la activación de PPAR- $\alpha$ aumenta la expresión de las apolipoproteínas A-I y A-II, lo que a su vez incrementa la síntesis y los niveles plasmáticos de HDL en humanos ${ }^{16-20}$.

Por este impacto favorable sobre el perfil lipídico sanguíneo, los fibratos han sido utilizados como una alternativa farmacológica para el control de la ateroesclerosis y sus complicaciones isquémicas. El estudio VA-HIT (Veterans Affairs High-density Lipoprotein Intervention Trial ${ }^{21}$, demostró el impacto favorable de la monoterapia con fibratos sobre la enfermedad cardiovascular en prevención 
secundaria en sujetos cuya principal anormalidad lipídica basal era la presencia de niveles bajos de c-HDL $(<40$ $\mathrm{mg} / \mathrm{dl}$ ). Por lo tanto, esta evidencia -junto a otros estudios previos- apoya el impacto favorable que exhibe el uso de los fibratos como monoterapia en el manejo del riesgo cardiovascular ateroesclerótico. Por otro lado, y en un contexto de terapia hipolipemiante combinada, el estudio ACCORD Lipid (Action to Control Cardiovascular Risk in Diabetes) $)^{22}$-adicionando fenofibrato en pacientes con diabetes tipo 2 (DM-2) que ya estaban recibiendo terapia con estatinas- evidenció que la terapia combinada aumentaba los niveles de c-HDL y disminuía los triglicéridos, aunque la incidencia de eventos cardiovasculares no fue distinta entre ambos grupos de estudio. Debido a estos resultados, actualmente existe controversia sobre el verdadero impacto beneficioso de los fibratos en la prevención de eventos cardiovasculares cuando se asocian a un tratamiento óptimo con estatinas. Sin embargo, un artículo reciente -que analizó post-hoc el subgrupo de pacientes con dislipidemia aterogénica (triglicéridos $\geq 204 \mathrm{mg} / \mathrm{dL}$ y c-HDL $\leq 34 \mathrm{mg}$ / dL) que fueron incluidos en los últimos grandes estudios con fibratos- demostró que este fármaco disminuiría significativamente el riesgo de eventos cardiovasculares en comparación con sujetos que no presentaban esta dislipidemia. ${ }^{23}$ Por lo tanto, el uso de este fármaco asociado a estatinas podría recomendarse en pacientes con dislipidemia aterogénica, una alteración bioquímica característica de los pacientes con DM-2 y síndrome metabólico.

Buscando avanzar en la comprensión de los efectos de los fibratos sobre el metabolismo del colesterol y la funcionalidad de las HDLs, el uso de modelos experimentales ofrecen una interesante alternativa de investigación. El ratón es ampliamente utilizado para estudios preclínicos de ateroesclerosis, a pesar de que el metabolismo de lípidos y lipoproteínas de esta especie muestra ciertas diferencias con respecto al ser humano. Por lo tanto, en este trabajo se propuso evaluar el impacto de la administración de fibratos sobre el metabolismo del colesterol HDL y la función antioxidante plasmática, usando el ratón como modelo experimental.

\section{Materiales y Métodos}

Animales y dietas. En este trabajo, se utilizaron ratones machos C57BL/6 silvestres (2-3 meses de edad), que fueron mantenidos en condiciones de temperatura y ciclos de luz controlados. Estos fueron alimentados con dieta control (ProLab RMH 3000, Labdiet) suplementada con 0,2\% de ciprofibrato (Sanofi) durante 7 días, con comida y agua disponibles ad libitum. Para los diferentes procedimientos descritos a continuación, los ratones fueron anestesiados mediante inyección intraperitoneal de ketamina:xilacina. Los protocolos de cuidado, manejo y procedimientos experimentales en los animales fueron aprobados previamente por el Comité de Ética y Bienestar Animal de la Escuela de Medicina de la Pontificia Universidad Católica de Chile.

Toma de muestras. Después de la inducción anestésica, los animales se sometieron a una fístula biliar para la recolección de bilis durante 30 minutos. Transcurrido este tiempo, se extrajo sangre por punción de la vena cava inferior. El plasma se obtuvo por centrifugación de la sangre a $4000 \mathrm{rpm}$ durante 20 minutos a $4^{\circ} \mathrm{C}$ y se almacenó a $-20^{\circ} \mathrm{C}$ para los análisis correspondientes. A continuación, los animales fueron eutanasiados por dislocación cervical y se removió el hígado, el cual fue congelado en nitrógeno líquido antes de almacenarlo a $-80^{\circ} \mathrm{C}$ para los análisis posteriores.

Fraccionamiento de las lipoproteínas plasmáticas y determinación del colesterol lipoproteico y colesterol, y triglicéridos plasmáticos totales. A partir del plasma, se realizó un fraccionamiento de las lipoproteínas plasmáticas mediante cromatografía líquida (FPLC) midiendo el contenido de colesterol en cada fracción, así como en el plasma total, por un ensayo enzimático descrito previamente ${ }^{24}$. La concentración plasmática de triglicéridos se determinó utilizando un kit comercial (Sigma).

Preparación de muestras de hígado y análisis de expresión proteica hepática por Western blot. A partir del tejido hepático, se prepararon extractos de membranas celulares totales que fueron sometidas a separación electroforética del contenido proteico en geles de acrilamida al 10-12\% seguido de transferencia a membranas de nitrocelulosa para análisis mediante inmunodetección por Western blot. Se utilizaron los anticuerpos contra SR-BI (anti-región carboxilo-terminal no comercial donado por el Dr. Monty Krieger, Massachusetts Institute of Technology, EE.UU.), ABCA1 (Novus), ABCG1 (Abcam), ABCG8 (donado por la Dra. Helen Hobbs, University of Texas, EE.UU.) y el control de carga $\varepsilon$-COP (también obtenido desde Massachusetts Institute of Technology, EE.UU).

Análisis del daño oxidativo plasmático. Usando $2 \mu \mathrm{L}$ de plasma en dilución 1:10, se separaron las proteínas plasmáticas por electroforesis y se transfirieron a nitrocelulosa -como se describió previamente- para inmunodetección de 


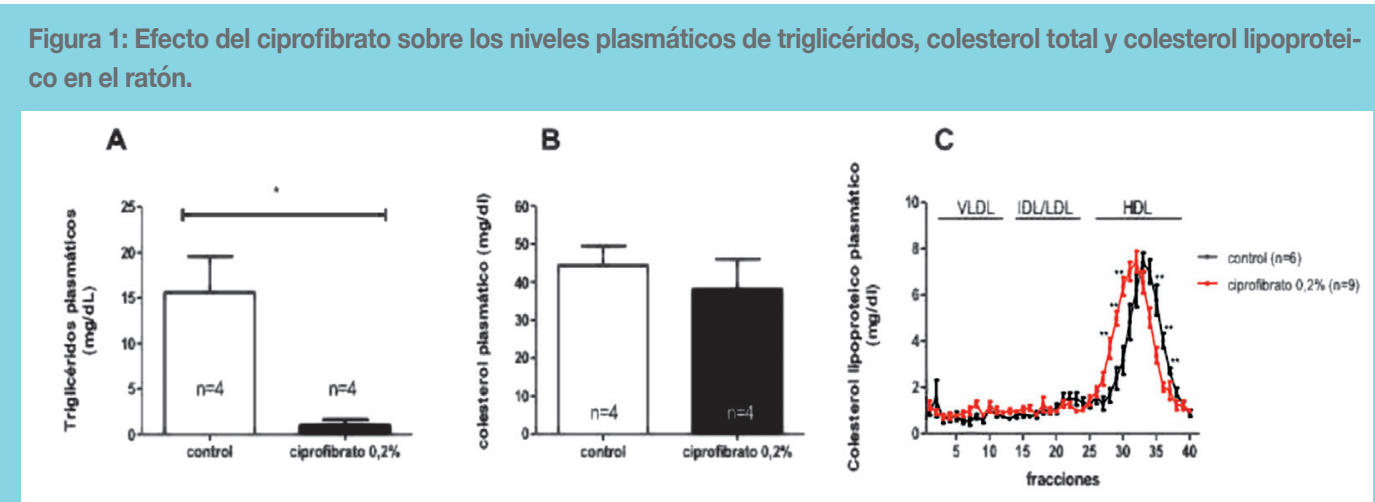

Los ratones C57BL/6 recibieron dieta control sin o con suplementación de ciprofibrato al 0,2\% durante 7 días. Tras este tiempo, se obtuvieron muestras sanguíneas para medir los niveles totales de triglicéridos (A) y colesterol plasmático (B) en ayunas. Las lipoproteínas plasmáticas fueron separadas por cromatografía líquida y las fracciones obtenidas posteriormente se sometieron a análisis de colesterol (C). * $p<0,05$; $* * p<0,01$.

epítopes oxidados. La determinación de daño oxidativo proteico se realizó con un anticuerpo contra nitrotirosina (Millipore), 4-hidroxinonenal (Abcam) y albúmina (Santa Cruz), este último como control de carga.

Medición del contenido de ADN hepático. Tras homogeneizar 50-100 mg de hígado en solución de Chomczynski con fenol-cloroformo, se precipitó el contenido de la fase orgánica con etanol, el cual fue procesado para cuantificación de ADN midiendo su absorbancia en un espectrofotómetro a $260 \mathrm{~nm}$.

Determinación del contenido de colesterol hepático. Tras homogeneizar 0,1-0,15 gramos de hígado, se realizó una extracción orgánica de Folch recuperando la fase clorofórmica para secado, resuspensión y posterior determinación del contenido de colesterol como fue descrito previamente.

Evaluación de la funcionalidad antioxidante del plasma. Este ensayo detecta la tasa de oxidación de dihidrorodamina (DHR) hacia rodamina fluorescente en ausencia o presencia de un volumen de plasma equivalente a $10 \mu \mathrm{g}$ de colesterol para cada muestra. La fluorescencia se midió cada 2 minutos durante 1 hora a $37^{\circ} \mathrm{C}$ a una longitud de onda de 485/538 nm excitación/emisión ${ }^{25}$, para luego calcular la tasa de oxidación de cada muestra expresada como unidades arbitrarias de fluorescencia (UAF)/min.

Análisis estadístico. Los resultados de las variables continuas se presentan como promedio \pm desviación estándar (DE) y se aplicó el test U de Mann-Whitney para la comparación de los resultados de datos no paramétricos usan- do el programa Graphpad. Las diferencias se consideraron estadísticamente significativas con $\mathrm{p}<0,05$.

Resultados: Para evaluar el impacto de los fibratos sobre el metabolismo del colesterol HDL y la funcionalidad de estas lipoproteínas de alta densidad, se utilizaron ratones machos C57BL/6 alimentados con dieta control o esta misma dieta suplementada con $0,2 \%$ de ciprofibrato durante 7 días. Tras este período, se tomaron muestras de bilis, sangre e hígado a partir de ambos grupos experimentales.

Tamaño hepático. Con respecto a la condición control, el tratamiento con ciprofibrato indujo hepatomegalia, evidenciada por un aumento en el peso absoluto (desde 1,3 $\pm 0,2$ g vs. $1,8 \pm 0,3 \mathrm{~g}, \mathrm{p}<0,0001)$ como consecuencia de una hipertrofia -sin hiperplasia- hepatocitaria, ya que el uso de fibratos incluso determinó una disminución significativa en el contenido de ADN hepático (5727 \pm 1422 ng ADN/mg hígado vs. $3770 \pm 1263$ ng ADN/mg hígado, $\mathrm{p}=0,0127$ ). Este incremento del tamaño hepático, inducido por el uso de fibratos, ha sido previamente reportado en los ratones, pero no ocurre en humanos.

Niveles de triglicéridos y colesterol plasmáticos totales y de colesterol lipoproteico. En muestras tomadas con condiciones de ayuno, el tratamiento con ciprofibrato disminuyó los niveles de triglicéridos plasmáticos $(15,6 \pm$ $3,97 \mathrm{mg} / \mathrm{dL}$ vs. $0,98 \pm 0,63 \mathrm{mg} / \mathrm{dL}, \mathrm{p}=0,0286$ ) (fig. $1 \mathrm{~A}$ ), pero no modificó los niveles de colesterol plasmático total $(44,3 \pm 5,2 \mathrm{mg} / \mathrm{dL}$ vs. 38,1 \pm 7,9 mg/dL, p=ns) con respecto al grupo control (fig. 1B). Para evaluar eventuales cambios en fracciones específicas de colesterol lipoproteico, las muestras de plasma fueron sometidas a fraccionamien- 
Figura 2: Efecto del ciprofibrato sobre los niveles proteicos hepáticos del receptor SR-BI y los transportadores ABCA1, ABCG1 y ABCG8 en el ratón.

A
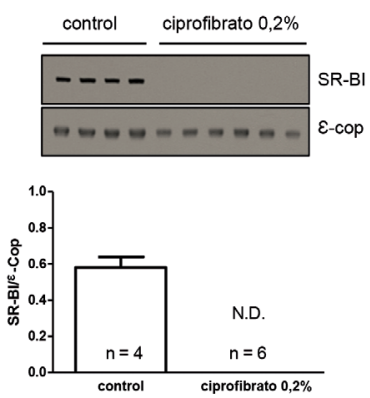

C
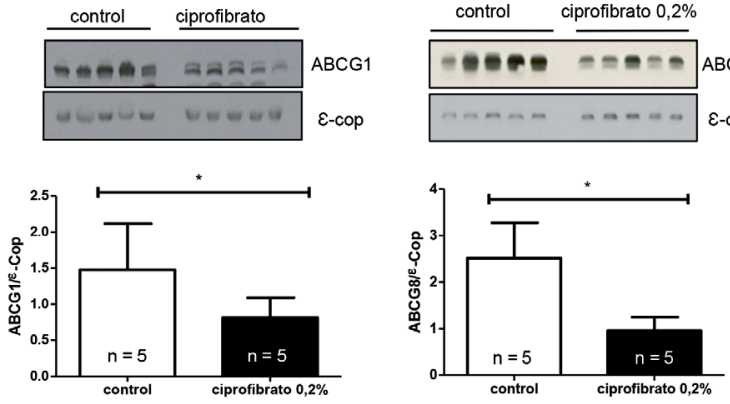

Los ratones C57BL/6 recibieron dieta control sin (barras blancas) o con ciprofibrato 0,2\% (barras negras) durante 7 días. Tras este período, se obtuvo tejido hepático para medir los niveles de SR-BI (A), ABCAl (B), ABCGI (C) y $A B C G 8(D)$ mediante Western blot. N.D.: no detectable. ${ }^{*} p<0,05$.

to cromatográfico seguido de medición del contenido de colesterol de cada fracción de elución. Como se muestra en la figura 1C, el peak de colesterol asociado a HDL se detectó entre las fracciones 27 y 39 en los ratones controles (curva de color negro), en cambio, el tratamiento con ciprofibrato mostró un peak de colesterol HDL desplazado hacia fracciones más precoces de la elución cromotográfica (desplazamiento hacia la izquierda en la curva de color rojo), indicando la presencia de partículas de HDL de mayor tamaño (fig. 1C). Consistente con la medición de colesterol plasmático total, el contenido de colesterol lipoproteico, calculado como la suma del área bajo la curva de cromatografía, no resultó diferente entre el grupo tratado con ciprofibrato versus el grupo control.

Niveles proteicos hepáticos del receptor de HDL y transportadores involucrados en el metabolismo de colesterol. Debido al papel que juega el receptor de HDL SR-BI en la captación selectiva de colesterol HDL hacia el hígado, se evaluó el efecto del uso de ciprofibrato sobre los niveles proteicos hepáticos de este receptor lipoproteico. Los resultados mostraron que los niveles proteicos hepáticos de SR-BI disminuyeron significativamente en los animales tratados con fibrato con respecto a su control (fig. 2A).

Del mismo modo, se estudiaron los niveles proteicos de transportadores de membrana relacionados con el eflujo de colesterol y la maduración de las partículas de HDL en el hígado. El transportador ABCA1, que promueve el eflujo de colesterol y fosfolípidos desde los hepatocitos hacia la apolipoproteína A-I pobre en lípidos, no se modificó por el tratamiento con fibrato ( $\mathrm{p}=\mathrm{ns}$ ) (fig. 2B). En el caso de ABCG1, el transportador que media el eflujo de colesterol desde las células hepáticas no parenquimatosas, mostró una disminución significativa de su expresión como consecuencia del tratamiento con fibratos ( $\mathrm{p}=0,0159)$ (fig. 2C). Como hemos mencionado, el transporte reverso de colesterol es uno de los mecanismos más conocidos que explicaría el efecto antiaterogénico de las HDL. Este proceso culmina con la excreción de colesterol desde el hígado hacia la bilis. Por lo tanto, adicionalmente evaluamos la expresión del transportador ABCG8, involucrado en la secreción biliar de colesterol. Con respecto al control, el tratamiento con fibrato disminuyó significativamente los niveles proteicos de ABCG8 ( $p=0,0159)$ (fig. 2D).

Contenido hepático de colesterol y secreción biliar de colesterol. Con respecto al grupo control, los hígados de los animales tratados con ciprofibrato presentaron niveles de colesterol disminuidos $(1,70 \pm 0,19 \mathrm{mg} / \mathrm{g}$ hígado vs $1,44 \pm 0,12 \mathrm{mg} / \mathrm{g}$ hígado, $\mathrm{p}=0,045)$.

Por otro lado, la concentración de colesterol en la bilis no se modificó por el tratamiento con fibrato $(14,7 \pm 4,5 \mathrm{mg} /$ dL vs. 18,8 $\pm 8,1 \mathrm{mg} / \mathrm{dL}, \mathrm{p}=\mathrm{ns}$ ) (fig. 3A), sin embargo, debido a un aumento en el flujo biliar $(p=0,0152)$ (fig. 3B), la secreción de colesterol biliar aumentó significativamente (desde $0,65 \pm 0,3 \mathrm{nmol} / \mathrm{min} / \mathrm{g}$ hígado a 1,04 \pm $0,36 \mathrm{nmol} / \mathrm{min} / \mathrm{g}$ hígado, $\mathrm{p}=0,0017)$ por el uso de ciprofibrato (fig. 3C).

Daño oxidativo y nitrosativo plasmático. Además del transporte de lípidos, las HDL -debido a su contenido de enzimas antioxidantes- modulan el estrés oxidativo, proceso que se produce por un aumento de las especies reactivas de oxígeno debido a una alteración del metabolismo celular o factores exógenos, provocando daño a nivel molecular (lípidos y proteínas) y celular. De esta forma, se estudió el impacto de la administración de fibratos sobre la nitrosilación en residuos de tirosina y la presencia de lí- 
Figura 3: Efecto del ciprofibrato sobre la concentración de colesterol biliar, flujo biliar y secreción de colesterol biliar en el ratón.

A

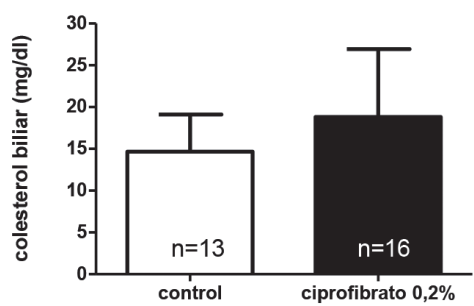

B

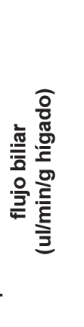

\section{C}

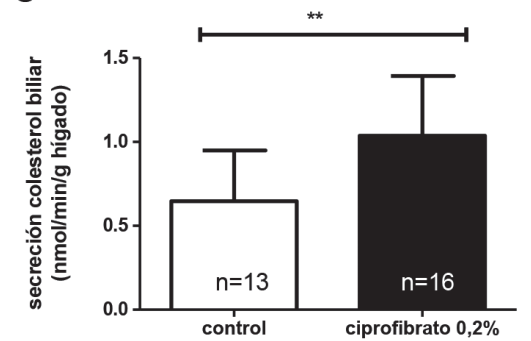

Los ratones C57BL/6 recibieron dieta control sin (barras blancas) o con ciprofibrato al 0,2\% (barras negras) durante 7 días. Tras este tiempo, se recolectó bilis para medir la concentración de colesterol biliar (A), el flujo biliar (B) y la secreción de colesterol biliar $(C)$. ${ }^{*} p<0,05 ; * * p<0,01$.

pidos peroxidados asociados a proteínas plasmáticas. Con respecto al control, el tratamiento con ciprofibrato aumentó los niveles de nitrotirosina ( $\mathrm{p}=0,0079)$ (fig. $4 \mathrm{~A}$ ), pero no modificó los de 4-hidroxinonenal $(\mathrm{p}=\mathrm{ns})$, asociados a proteínas plasmáticas (fig. 4B).

Funcionalidad antioxidante plasmática. Por otro lado, las partículas de HDL determinan en forma importante la funcionalidad antioxidante del plasma. Así, analizamos la capacidad antioxidante del plasma mediante un ensayo que detecta la oxidación de dihidrorodamina (DHR) a rodamina fluorescente en el tiempo. Usando este método, la fluorescencia de rodamina aumenta a medida que transcurre el ensayo, sin embargo, el contenido de antioxidantes del plasma será capaz de atenuar la oxidación espontánea del fluoróforo y, por tanto, disminuirá la pendiente de formación del producto oxidado. Comparando la capacidad antioxidante, el plasma total de los ratones alimentados con dieta suplementada con ciprofibrato mostró una pendiente en la curva de formación de rodamina significativamente menor con respecto al grupo control (13,9 vs. 22,4 UAF de rodamina/min, p<0,001, modelo lineal-varianza univariante), lo que indica que el tratamiento con este hipolipemiante mejora la capacidad antioxidante del plasma en el ratón (fig. 5).

Discusión: El presente estudio propuso evaluar el efecto de los fibratos sobre el metabolismo del colesterol HDL y la funcionalidad de estas lipoproteínas usando al ratón como modelo experimental de trabajo.

Consistente con lo descrito previamente en roedores, los resultados muestran que el tratamiento con fibrato en ratones produjo hepatomegalia, lo cual se explica por hipertrofia -sin hiperplasia- hepatocelular como consecuencia de un efecto proliferativo perixosomal reportado previamente en ratones ${ }^{20,26}$. Esto se debe a que los fibratos son activadores del receptor nuclear PPAR- $\alpha$, el cual estimula la transcripción de genes involucrados en el metabolismo de

Figura 4: Efecto del ciprofibrato sobre daño oxidativo proteico plasmático en el ratón.
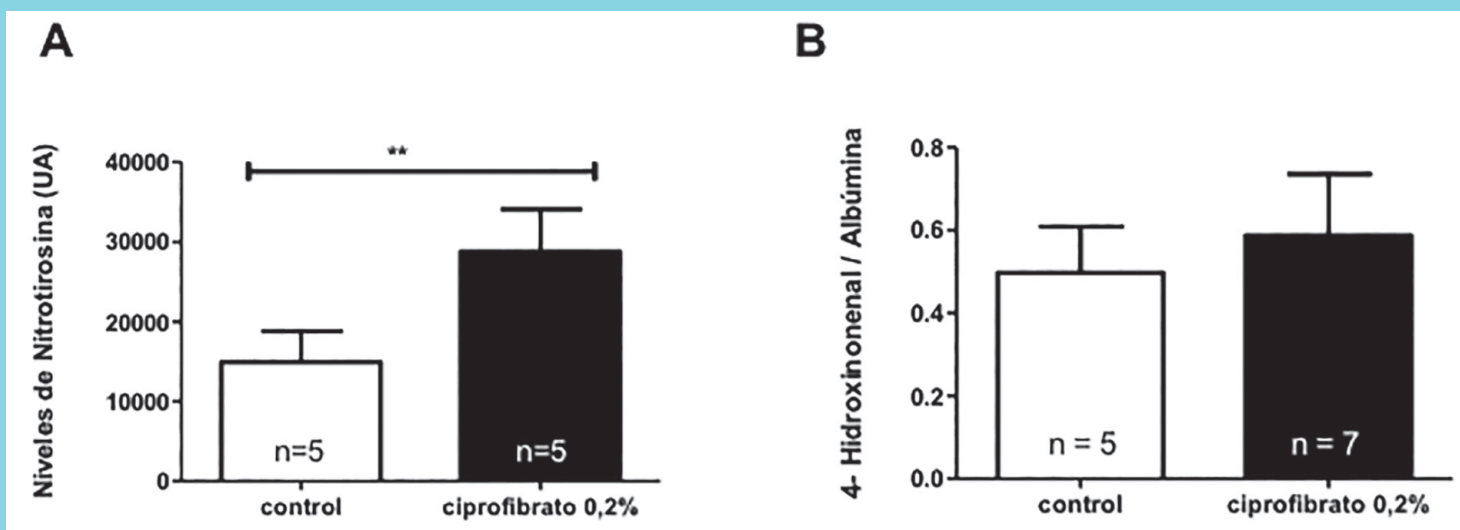

Los ratones C57BL/6 recibieron dieta control sin (barras blancas) o con ciprofibrato al 0,2\% (barras negras) durante 7 días. Tras este tiempo, se tomaron muestras sanguíneas para medir marcadores de daño oxidativo (nitrotirosina (A) y 4-hidroxinonenal (B)) en plasma mediante Western blot. ** $p<0,01$. 
Figura 5: Efecto del ciprofibrato sobre la capacidad antioxidante plasmática en el ratón.

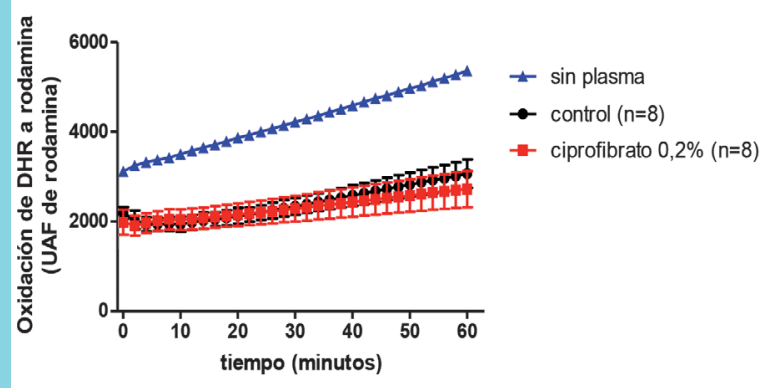

Los ratones C57BL/6 recibieron dieta control sin o con ciprofibrato al $0,2 \% \mathrm{du}$ rante 7 días. Tras este tiempo, se obtuvieron muestras sanguíneas para medir la oxidación de DHR hacia rodamina fluorescente durante 1 hora en ausencia de plasma (color azul) o la presencia de plasma obtenido de ratones alimentados con dieta control (color negro) o suplementada con ciprofibrato (color rojo).

lípidos (p.e., $\beta$-oxidación de ácidos grasos) presentes en los peroxisomas de los hepatocitos de los roedores ${ }^{27}$. En relación a este resultado, es importante destacar que este efecto no ha sido observado en humanos.

Característicamente, los fibratos estimulan la $\beta$-oxidación de ácidos grasos y la lipólisis de lipoproteínas ricas en triglicéridos, de modo que se esperaba que el tratamiento con ciprofibrato disminuyera los niveles de triglicéridos plasmáticos en los ratones. En contraste, los niveles de colesterol plasmático total no se modificaron por el uso de fibratos, aunque se observó un aumento en el tamaño, pero no en el contenido de colesterol, de las partículas HDL. En roedores, se ha descrito que los fibratos incluso disminuyen los niveles de c-HDL pues reducen la expresión de sus componentes apolipoproteicos apoA-I y apo A-II ${ }^{28}$, contrario a lo que ocurre en humanos, debido -en el caso de la apo A-I- a una variante nucleotídica en el promotor del gen correspondiente, lo que indica, la existencia de una regulación especie-específica de esta apolipoproteína por efecto de esta clase de hipolipemiantes ${ }^{29}$.

Los niveles proteicos hepáticos del receptor de HDL SRBI fueron suprimidos por el tratamiento con fibrato, lo que se correlaciona con el aumento en el tamaño de las partículas de HDL secundario a una menor captación selectiva hepática de colesterol mediada por este receptor. Las modificaciones observadas en el perfil de colesterol lipoproteico y en los niveles de SR-BI en el hígado del ratón ya habían sido previamente descritas y publicadas por nuestro laboratorio ${ }^{30}$. En ratones, los fibratos también disminuyen la expresión de la proteína de transferencia de fosfolípidos (PLTP) ${ }^{31}$, que media la transferencia de fosfolípidos desde VLDL a HDL, lo que también podría contribuir al remodelamiento de partículas de HDL. Debido a la expresión prácticamente indetectable de SR-BI, habríamos esperado un aumento significativo en los niveles de c-HDL y -eventualmente- un mayor incremento en el tamaño de estas partículas lipoproteicas. Sin embargo, el ciprofibrato actúa como activador de un factor transcripcional, de modo que puede plantearse la modulación de varios otros genes (apo A-I, apo A-II, apo C-III, lipasa lipoproteica, PLTP, ABCA1 y ABCG1) que regulan en el metabolismo de HDL lo que estaría determinando efectos compensatorios (p.e., menor síntesis y/o mayor catabolismo de HDL) sobre el dramático impacto detectado en los niveles proteicos de SR-BI en el hígado. Para evaluar esta proposición, medimos los niveles proteicos de los transportadores ABCA1 y ABCG1 hepáticos: el primero está involucrado en la biogénesis de HDL nacientes y la mantención de los niveles de HDL plasmáticos, mientras el segundo contribuye al eflujo de colesterol desde células periféricas y células hepáticas no parenquimatosas durante la maduración de las partículas de HDL. El tratamiento con fibratos disminuyó los niveles proteicos hepáticos de ABCG1, pero no ABCA1. Estos resultados indicarían que la maduración hepática de las partículas de HDL estaría disminuida por el uso de fibratos, lo que compensaría la disminución del catabolismo selectivo del colesterol de HDL debido a la ausencia de SR-BI, explicando así que los niveles de c-HDL plasmáticos no se modificaran en forma neta por este tratamiento.

El transporte reverso de colesterol es una de las funciones claves y más conocidas de las lipoproteínas HDL. Como consecuencia de este proceso, el colesterol es removido desde los tejidos periféricos, incorporado en esta clase lipoproteica, transportado en la circulación, entregado al hígado por vía directa o indirecta -dependiendo de la especie- y finalmente es excretado por la bilis. El transportador ABCG8, expresado en la superficie canalicular de los hepatocitos junto con ABCG5, es el encargado de la secreción de colesterol desde el hígado hacia la bilis. Con el uso de fibratos, los niveles proteicos hepáticos de ABCG8 disminuyeron significativamente, aunque el contenido de colesterol biliar no se modificó e incluso se detectó un aumento en la tasa neta de secreción biliar de colesterol como consecuencia de un incremento significativo en el flujo biliar. Debido a que la principal fuerza motora del flujo biliar y de la secreción de colesterol y fosfolípidos biliares es la secreción de sales biliares, el efecto estimulador del ciprofibrato sobre la secreción biliar podría explicarse por una inducción de la expresión hepática del transportador sinusoidal (NTCP) y/o del transportador canalicular 
BSEP (Biliary Salt Export Pump) de sales biliares lo que facilitaría el flujo transhepático de sales biliares ${ }^{32}$.

Considerando integralmente los resultados discutidos anteriormente a nivel hepático, el potente efecto del tratamiento con fibrato sobre la supresión de la expresión de SR-BI, que disminuye la captación de colesterol HDL hacia el hígado, y el aumento de la secreción del colesterol hacia la bilis podrían explicar la disminución en el contenido de colesterol hepático observado en los ratones tratados con esta clase de hipolipemiante. De hecho, estudios previos $^{33}$ han demostrado que el uso de bezafibrato también determina una reducción en los niveles de colesterol hepático en asociación con una inducción de SREBP-2, el factor transcripcional clave en el control de la expresión de HMGCoA reductasa, la enzima limitante en la síntesis de colesterol.

Además del transporte de lípidos, las partículas de HDL -debido a su contenido de varias enzimas antioxidantes- modulan el estrés oxidativo, proceso que refleja el desbalance entre la formación de agentes oxidantes y las defensas antioxidantes endógenas ${ }^{34}$. De esta forma, bajos niveles de especies reactivas de oxígeno (ROS) son esenciales para el funcionamiento normal de las células y la preservación de la funcionalidad de biomoléculas y complejos polimoleculares extracelulares, mientras que su aumento suprafisiológico gatilla modificaciones oxidativas a nivel mono- y polimolecular (lípidos, proteínas, lipoproteínas) y celular. Además, la interacción de ROS y óxido nítrico, un potente regulador del tono vascular, entre otras funciones, determina la producción de especies reactivas de nitrógeno (RNS) que potencian el daño molecular y celular como consecuencia de nitrosilaciones ${ }^{35}$. Tanto la oxidación como la nitrosilación de proteínas y lípidos han surgido como marcadores de daño oxidativo y se detectan -e incluso tendrían un papel causal- en una amplia variedad procesos patológicos, incluyendo las enfermedades crónicas no transmisibles ${ }^{35}$. En nuestro estudio, el ciprofibrato aumentó significativamente los niveles plasmáticos de nitrotirosina, indicando que la administración de esta droga en ratones silvestres generó estrés nitrosativo. Sin embargo, no observamos modificaciones en los niveles de 4-hidroxinonenal, producto de oxidación lipídica asociado a proteínas plasmáticas, indicando que este fibrato no tiene efecto en el daño oxidativo a lípidos, aunque no puede descartarse un resultado falso negativo dependiente de la calidad del anticuerpo utilizado. De hecho, Nakajima et al., ${ }^{36}$ han reportado que la activación de PPAR- $\alpha$ resulta en la sobreproducción de ROS en el músculo y el hígado de ratones tratados con fibratos y daño oxidativo dependiente de 4-hidroxinonenal como consecuencia de la inducción de varios genes que codifican enzimas generadoras de ROS como AOX y CYP4A y que son regulados por PPAR- $\alpha$.

Por otro lado, la medición de la capacidad antioxidante evidenció que el plasma de animales alimentados con dieta suplementada con fibrato tenía mayor capacidad antioxidante que su control respectivo. A primera vista, este resultado parece contradictorio con los hallazgos de mayor estrés oxidativo plasmático demostrado para el uso de fibratos. Sin embargo, los fibratos -como activadores de factores transcripcionales como PPAR- $\alpha$ tienen la posibilidad de ejercer efectos pleiotrópicos y, por tanto, no descartamos que su administración pueda estar ejerciendo efectos inductores en algunas enzimas antioxidantes asociadas a las partículas de HDL, aunque finalmente el balance entre la generación de estrés oxidativo y la estimulación de defensa antioxidante dependiente de HDL sea negativo a favor de un daño nitrosativo de las proteínas plasmáticas. Tampoco se puede descartar que el efecto primario del ciprofibrato sea la inducción de estrés oxidativo, lo cual gatilla mecanismos compensatorios fibrato-independientes -como una mayor actividad antioxidante de HDL- tendientes a contrarrestar el ambiente pro-oxidante inducido por esta clase de hipolipemiantes en el ratón.

Los resultados de este estudio han permitido obtener nueva información sobre el efecto metabólico y funcional de la administración de fibratos en ratones, lo cual podría ayudar comprender los resultados de estudios clínicos recientes que han usado esta clase de hipolipemiantes en humanos.

Agradecimientos: Este trabajo fue financiado por el proyecto regular $\mathrm{N}^{\mathrm{o}} 1110712$ del Fondo Nacional de Desarrollo Científico y Tecnológico - Chile. 


\section{Referencias}

1. YUSUF S, REDDY S, OUNPUU S AS. Global Burden of Cardiovascular Diseases part I: General considerations, the Epidemiologic Transition, Risk Factors, and Impact of Urbanization. Circulation. 2001;104:2746-2753.

2. YUSUF S, REDDY S, OUNPUU S AS. Global Burden of Cardiovascular Diseases part II: Variations in Cardiovascular Disease by Specific Ethnic Groups and Geographic Regions and Prevention Strategies. Circulation. 2001;104:2855-2864.

3. YUSUF S, HAWKEN S, OUNPUU S, DANS T, AVEZUM A, LANAS F, et al. Effect of potentially modifiable risk factors associated with myocardial infarction in 52 countries (the INTERHEART study): case-control study. Lancet. 2004;364:937-952.

4. GRUNDY SM, CLEEMAN JI, MERZ CN, BREWER HB JR, CLARK LT, HUNNINGHAKE DB, et al. Implications of recent clinical trials for the National Cholesterol Education Program Adult Treatment Panel III guidelines. Circulation. 2004;110:227-239.

5. LINK J, ROHATGI A, DE LEMOS J. HDL Cholesterol: Physiology, Pathophysiology, and Management. Curr Probl Cardiol. 2007;32:268-314.

6. CHOI BG, VILAHUR G, YADEGAR D, VILES-GONZALEZ JF, BADIMON JJ. The role of high-density lipoprotein cholesterol in the prevention and possible treatment of cardiovascular diseases. Curr Mol Med. 2006;6:571-587.

7. SINGH I, SHISHEHBOR M AB. High-Density Lipoprotein as a Therapeutic Target. A systematic review. JAMA. 2007;298:786-798.

8. TOTH PP. HIGH-density lipoprotein as a therapeutic target: clinical evidence and treatment strategies. Am J Cardiol. 2005;96:50K - 58K; discussion 34K - 35K.

9. Encuesta Nacional de Salud ENS Chile 2009-2010. Ministerio de Salud de Chile.

10. ACTON S, RIGOTTI A, LANSCHULZ KT, XU S, HOBBS HH, KRIEGER M. Identification of scavenger receptor SRBI as a high density lipoprotein receptor. Science (80- ). 1996;271:518-520.
11. FIELDING CJ, FIELDINST PE. Molecular physiology of reverse cholesterol transport. J Lipid Res. 1995;36:211-228.

12. ZANONI P, KHETARPAL SA, LARACH DB, HANCOCK-CERUTTI WF, MILLAR JS, CUCHEL M, et al. Rare variant in scavenger receptor BI raises HDL cholesterol and increases risk of coronary heart disease. Science. 2016;351:1166-1171.

13. HAFIANE A, GENEST J. HDL, atherosclerosis, and emerging therapies. Cholesterol. 2013; 2013: 891403

14. GORDON SM, HOFMANN S, ASKEW DS, DAVIDSON WS. High density lipoprotein: it's not just about lipid transport anymore. Trends Endocrinol Metab. 2011; 22: 9-15.

15. RADER DJ, HOVINGH GK. HDL and cardiovascular disease. Lancet. 2014; 384: 618-25

16. KERSTEN S. Peroxisome proliferator activated receptors and lipoprotein metabolism. PPAR Res. 2008; 2008: 1-11.

17. TENENBAUM A, FISMAN EZ. Fibrates are an essential part of modern anti-dyslipidemic arsenal: spotlight on atherogenic dyslipidemia and residual risk reduction. Cardiovasc Diabetol. 2012; 11: 125 .

18. BERGER JP, AKIYAMA TE, MEINKE PT. PPARs: Therapeutic targets for metabolic disease. Trends Pharmacol Sci. 2005; 26: 244-251.

19. KERSTEN S, DESVERGNE B, WAHLI W. Roles of PPARs in health and disease. Nature. 2000; 405: 421-424.

20. FRUCHART JC, STAELS B, DURIEZ P. The role of fibric acids in atherosclerosis. Curr Atheroscler Rep. 2001; 3: 8392.

21. ROBINS SJ, COLLINS D, WITTES JT, PAPADEMETRIOU V, DEEDWANIA PC, SCHAEFER EJ, et al. Relation of Gemfibrozil Treatment and Lipid Levels With Major Coronary Events. JAMA. 2001; 285: 1585-1591.

22. GINSBERG HN, MARSHALL BE, LOVATO LC, CROUSE III JR, LEITER LA, LINZ P, FRIEDEWALD WT, et al. Effects of Combination Lipid Therapy in Type 2 Diabetes Me- 
23. SACKS FM, CAREY VJ FJ-C. Correspondence on Combination Lipid Therapy in Type 2 Diabetes. N Engl J Med. 2010; 363: 692-694.

24. AlLAIN C, POON L, CHAN C, RICHMOND W, FU P. Enzymatic Determination of Total Serum Cholesterol. Clin Chem. 1974; 20: 470-475.

25. KELESIDIS T, CURRIER JS, HUYNH D, MERIWETHER D, CHARLES-SCHOEMAN C, REDDY ST, et al. A biochemical fluorometric method for assessing the oxidative properties of HDL. J Lipid Res. 2011; 52: 2341-2351.

26. REDDY J, QURESHI S. Tumorigenicity of the hypolipidaemic perixosome proliferator ethyl-alpha-p-chlorophenoxyisobutyrate (clofibrate) in rats. Br J Cancer. 1979; 40: 476-482.

27. LAZAROW PB. Rat liver perixosomes catalyze the beta oxidation of fatty acids. J Biol Chem. 1978; 253: 1522-1528.

28. STAELS B, VAN TOL A, ANDREU T, AUWERX J. Fibrates influence the expression of genes involved in lipoprotein metabolism in a tissue-selective manner in the rat. Arter Thromb. 1992; 12: 286-294.

29. STAELS B, AUWERX J. Regulation of apo A-I gene-expression by fibrates. Atherosclerosis. 1998; 137: S19-S23.

30. MARDONES P, PILON A, BOULY M, DURAN D, NISHIMOTO T, ARAI H, et al. Fibrates Down-regulate Hepatic Scavenger Receptor Class B Type I Protein Expression in
31. BOULY M, MASSON D, GROSS B, JIANG XC, FIEVET $\mathrm{C}, \mathrm{CASTRO} \mathrm{G}$, et al. Induction of the phospholipid transfer protein gene accounts for the high density lipoprotein enlargement in mice treated with fenofibrate. J Biol Chem. 2001; 276: 25841-25847.

32. KOK T, BLOKS VW, WOLTERS H, HAVINGA R, JANSEN PL, STAELS B, et al. Peroxisome proliferator-activated receptor alpha (PPARalpha)-mediated regulation of multidrug resistance 2 (Mdr2) expression and function in mice. Biochem J. 2003; 369: 539-547.

33. NAKAJIMA T, TANAKA N, SUGIYAMA E, KAMIJO Y, HARA A, HU R, et al. Cholesterol-lowering effect of bezafibrate is independent of peroxisome proliferator-activated receptor activation in mice. Biochem Pharmacol. 2008; 76: 108-119.

34. PELUFFO G, RADI R. Biochemistry of protein tyrosine nitration in cardiovascular pathology. Cardiovasc Res. 2007; 75: 291-302.

35. HSIEH H-J, LIU C-A, HUANG B, TSENG AH, WANG DL. Shear-induced endothelial mechanotransduction: the interplay between reactive oxygen species (ROS) and nitric oxide (NO) and the pathophysiological implications. J Biomed Sci. 2014; $21: 3$.

36. NAKAJIMA T, TANAKA N, LI G, et al. Effect of bezafibrate on hepatic oxidative stress: comparison between conventional experimental doses and clinically-relevant doses in mice. Redox Rep. 2010;15:123-130. 